Management of Microbial Resources in the Environment 

Abdul Malik - Elisabeth Grohmann

Madalena Alves

Editors

\section{Management of Microbial Resources in the Environment}

望 Springer 


\section{Editors}

Abdul Malik

Department of Agricultural Microbiology

Faculty of Agricultural Sciences

Aligarh Muslim University

Aligarh, India

\section{Visiting Professor/Scientist \\ Division of Infectious Diseases \\ University Medical Center Freiburg \\ Freiburg, Germany}

\author{
Madalena Alves \\ Center of Biological Engineering \\ University of Minho \\ Braga, Portugal
}

\author{
Elisabeth Grohmann \\ Division of Infectious Diseases \\ University Medical Center Freiburg \\ Freiburg, Germany
}

ISBN 978-94-007-5930-5

ISBN 978-94-007-5931-2 (eBook)

DOI 10.1007/978-94-007-5931-2

Springer Dordrecht Heidelberg New York London

\author{
Library of Congress Control Number: 2013933022
}

(C) Springer Science+Business Media Dordrecht 2013

This work is subject to copyright. All rights are reserved by the Publisher, whether the whole or part of the material is concerned, specifically the rights of translation, reprinting, reuse of illustrations, recitation, broadcasting, reproduction on microfilms or in any other physical way, and transmission or information storage and retrieval, electronic adaptation, computer software, or by similar or dissimilar methodology now known or hereafter developed. Exempted from this legal reservation are brief excerpts in connection with reviews or scholarly analysis or material supplied specifically for the purpose of being entered and executed on a computer system, for exclusive use by the purchaser of the work. Duplication of this publication or parts thereof is permitted only under the provisions of the Copyright Law of the Publisher's location, in its current version, and permission for use must always be obtained from Springer. Permissions for use may be obtained through RightsLink at the Copyright Clearance Center. Violations are liable to prosecution under the respective Copyright Law.

The use of general descriptive names, registered names, trademarks, service marks, etc. in this publication does not imply, even in the absence of a specific statement, that such names are exempt from the relevant protective laws and regulations and therefore free for general use.

While the advice and information in this book are believed to be true and accurate at the date of publication, neither the authors nor the editors nor the publisher can accept any legal responsibility for any errors or omissions that may be made. The publisher makes no warranty, express or implied, with respect to the material contained herein.

Printed on acid-free paper

Springer is part of Springer Science+Business Media (www.springer.com) 


\section{Preface}

Microorganisms are microscopic forms of life that include bacteria, protozoa, algae, fungi and viruses, and comprise the greatest number of individual organisms on the earth. At best, however, science has identified and is aware of only a minute fraction of them. Somewhere between 0.1 and $1.0 \%$ of all bacterial species have been described and the vast majority of the remaining bacterial species is unknown. Uncultured organisms comprise the vast majority of the microbial world. Although culturing has been indispensable for increasing our understanding of specific organisms, problems with using culturing for community analysis arise from the fact that an artificial homogenous medium typically allows growth of only a small fraction of the organisms. Culturing fails to reproduce the ecological niches and symbiotic relationships encountered in complex natural environments that are required to support the full spectrum of microbial diversity. Microorganisms are valuable genetic resources, which contribute to the global economic and social development. Microbial Resources include the exploration, collection, characterization, evaluation and conservation of microbes for sustainable utilization in the development of the global/national economy, i.e. Agriculture, Ecosystem, Environment, Industry and Medicine. Microbes play a critical role in natural biogeochemical cycles and make up about $60 \%$ of the Earth's biomass. Many research institutes/universities all over the world are carrying out microbiological and biotechnological research, which results in generating lots of genomic resources like cDNA libraries, gene constructs, promoter regions, transgenes etc. These are valuable resources for gene discovery and transgenic product development.

In this book we provide up-to-date information about the management of microbial resources in the environment. This book covers the ecology of microorganisms in natural and engineered environments; genomic, metagenomic and molecular advances in understanding of microbial interactions; microbial diversity and phylogeny; ecological studies of human, animal and plant microbiology and disease; microbial processes and interactions in the environment; and technological developments. This book is not intended to serve as an encyclopedic review of the subject. However, 
the various chapters incorporate both theoretical and practical aspects and may serve as baseline information for future research through which significant development is possible.

The book has twenty chapters, with each focused on a specific topic to cover diverse perspective topics. An introductory chapter on management of microbial resources in the environment is included. Topics include: recent development in the methods of studying microbial diversity, microbial resource centers towards harnessing microbial diversity for human welfare, polyphasic identification and preservation of fungal diversity, fungal biodiversity: a potential tool in plant disease management, bioinformatics approaches in studying microbial diversity, recent advances in metagenomic studies of soil microbial communities, mobile genetic elements (MGEs) carrying catabolic genes, conjugative plasmids in anthropogenic soils, potential eco-friendly soil microorganisms, current aspects of metal resistant bacteria in bioremediation, anaerobic digestion of the organic fraction of municipal solid waste, microbial insecticides: food security and human health, microbes (PGPR) in sustainable agriculture, antibiotic resistance gene pool and bacterial adaptation to xenobiotics in the environment, synthetic lethal genetic interaction networks and their utility for anticancer therapy, adaptation of Candida albicans for growth within the host, role of marine anaerobic bacteria and archaea in bioenergy production, bacteriocins: natural weapons for control of food pathogens, and anaerobic degradation of lindane and other $\mathrm{HCH}$ isomers.

With great pleasure, we extend our sincere thanks to all our well-qualified and internationally renowned contributors from different countries for providing the important, authoritative and cutting edge scientific information/technology to make this book a reality. All chapters are well illustrated with appropriately placed tables and figures and enriched with up to date information. We are also thankful to the reviewers who carefully and timely reviewed the manuscripts. Dr. Abdul Malik is also thankful to the Department of Biotechnology, Govt. of India, New Delhi for DBT CREST award/Fellowship during the preparation of the book.

We are extremely thankful to Springer, Dordrecht, the Netherlands for completing the review process expeditiously to grant acceptance for publication. We appreciate the great efforts of the book publishing team, especially of Dr. Alexandrine Cheronet, Senior Publishing Editor Environmental Sciences in responding to all queries very promptly.

We express sincere thanks to our family members for all the support they provided, and regret the neglect and loss they suffered during the preparation of this book.

Aligarh, India

Freiburg, Germany

Braga, Portugal
Abdul Malik

Elisabeth Grohmann

Madalena Alves 


\section{Contents}

1 Management of Microbial Resources in the Environment:

A Broad Perspective

Abdul Malik, Farhana Masood, and Elisabeth Grohmann

2 Retracted: Recent Development in the Methods of Studying Microbial Diversity

Mohd Ikram Ansari and Abdul Malik

3 Microbial Resource Centers Towards Harnessing Microbial

Diversity for Human Welfare

Showkat Ahmad Lone, Abdul Malik, and Jasdeep Chatrath Padaria

4 Fungal Biodiversity: A Potential Tool in Plant

Disease Management

Shabbir Ashraf and Mohammad Zuhaib

5 Polyphasic Identification and Preservation of Fungal Diversity:

Concepts and Applications

Marta F. Simões, Leonel Pereira, Cledir Santos, and Nelson Lima

6 Bioinformatics Approaches in Studying Microbial Diversity

Mohammad Tabish, Shafquat Azim, Mohammad Aamir Hussain,

Sayeed Ur Rehman, Tarique Sarwar, and Hassan Mubarak Ishqi

7 Recent Advances and Perspectives in Metagenomic Studies of Soil Microbial Communities

E.V. Pershina, E.E. Andronov, A.G. Pinaev, and N.A. Provorov

8 Mobile Genetic Elements (MGEs) Carrying Catabolic Genes

Masaki Shintani and Hideaki Nojiri

9 Conjugative Plasmids in Anthropogenic Soils

Elisabeth Grohmann 
10 Potential Eco-friendly Soil Microorganisms: Road Towards

Green and Sustainable Agriculture.

Surinder Kaur, Gurpreet Singh Dhillon, Satinder Kaur Brar,

Vijay Bahadur Chauhan, Ramesh Chand, and Mausam Verma

11 Current Aspects of Metal Resistant Bacteria in Bioremediation:

From Genes to Ecosystem.

Farhana Masood and Abdul Malik

12 Anaerobic Digestion of the Organic Fraction of Municipal Solid Waste

Muna Albanna

13 Microbial Insecticides: Food Security and Human Health

M. Shafiq Ansari, Salman Ahmad, Nadeem Ahmad, Tufail Ahmad, and Fazil Hasan

14 Plant Growth Promoting Rhizobacteria (PGPR):

Microbes in Sustainable Agriculture.

Jay Shankar Singh and D.P. Singh

15 Retracted: Antibiotic Resistance Gene Pool

and Bacterial Adaptation to Xenobiotics in the Environment

Mohd Ikram Ansari and Abdul Malik

16 Synthetic Lethal Genetic Interaction Networks and Their

Utility for Anticancer Therapy

Saman Khan, Amit Kumar Sonkar, and Shakil Ahmed

17 Adaptation of Candida albicans for Growth Within the Host

Zuraini Zakaria, Basma Rajeh Mohammad Abu Arra, and Sumathi Ganeshan

18 The Role of Marine Anaerobic Bacteria and Archaea in Bioenergy Production

A.J. Cavaleiro, A.A. Abreu, D.Z. Sousa, M.A. Pereira, and M.M. Alves

19 Bacteriocins: Natural Weapons for Control of Food Pathogens

Nabil Ben Omar, Hikmate Abriouel, Ismail Fliss, Miguel Ángel

Ferandez-Fuentes, Antonio Galvez, and Djamel Drider

20 Anaerobic Degradation of Lindane and Other HCH Isomers.

Farrakh Mehboob, Alette A.M. Langenhoff, Gosse Schraa, and Alfons J.M. Stams

Retraction Note to

Index 


\section{Contributors}

A.A. Abreu IBB - Institute for Biotechnology and Bioengineering, Centre of Biological Engineering, University of Minho, Braga, Portugal

Hikmate Abriouel Área de Microbiología, Departamento de Ciencias de la Salud, Facultad de Ciencias Experimentales, Edif. B3, Universidad de Jaén, Jaén, Spain

Nadeem Ahmad Department of Plant Protection, Faculty of Agricultural Sciences, Aligarh Muslim University, Aligarh, India

Salman Ahmad Department of Plant Protection, Faculty of Agricultural Sciences, Aligarh Muslim University, Aligarh, India

Tufail Ahmad Department of Plant Protection, Faculty of Agricultural Sciences, Aligarh Muslim University, Aligarh, India

Shakil Ahmed Molecular and Structural Biology Division, CSIR - Central Drug Research Institute, Lucknow, India

Muna Albanna Water and Environmental Engineering Department, School of Natural Resources Engineering, German Jordanian University, Amman, Jordan

M.M. Alves IBB - Institute for Biotechnology and Bioengineering, Centre of Biological Engineering, University of Minho, Braga, Portugal

E.E. Andronov All-Russia Research Institute for Agricultural Microbiology, Saint-Petersburg, Russia

M. Shafiq Ansari Department of Plant Protection, Faculty of Agricultural Sciences, Aligarh Muslim University, Aligarh, India

Mohd Ikram Ansari Department of Agricultural Microbiology, Faculty of Agricultural Sciences, Aligarh Muslim University, Aligarh, India

Basma Rajeh Mohammad Abu Arra Biology Programme, School of Distance Education, Universiti Sains Malaysia, Minden, Penang, Malaysia 
Shabbir Ashraf Department of Plant Protection, Faculty of Agriculture Sciences, Aligarh Muslim University, Aligarh, Uttar Pradesh, India

Shafquat Azim Department of Biochemistry, Faculty of Life Sciences, Aligarh Muslim University, Aligarh, Uttar Pradesh, India

Satinder Kaur Brar INRS-ETE, Université du Québec, Québec, Canada

A.J. Cavaleiro IBB - Institute for Biotechnology and Bioengineering, Centre of Biological Engineering, University of Minho, Braga, Portugal

Ramesh Chand Department of Mycology \& Plant Pathology, Institute of Agricultural Sciences, Banaras Hindu University (BHU), Varanasi, Uttar Pradesh, India

Vijay Bahadur Chauhan Department of Mycology \& Plant Pathology, Institute of Agricultural Sciences, Banaras Hindu University (BHU), Varanasi, Uttar Pradesh, India

Gurpreet Singh Dhillon INRS-ETE, Université du Québec, Québec, QC, Canada

Djamel Drider Laboratoire des Procédés Biologiques, Génie Enzymatique et Microbien (ProBioGEM), UPRES-EA 1026, Polytech'Lille/IUTA, Université Lille Nord de France, Villeneuve d'Ascq Cedex, France

Miguel Ángel Ferandez-Fuentes Área de Microbiología, Departamento de Ciencias de la Salud, Facultad de Ciencias Experimentales, Edif. B3, Universidad de Jaén, Jaén, Spain

Laboratoire des Procédés Biologiques, Génie Enzymatique et Microbien (ProBioGEM), UPRES-EA 1026, Polytech'Lille/IUTA, Université Lille Nord de France, Villeneuve d'Ascq Cedex, France

Ismail Fliss STELA Dairy Research Center, Nutraceuticals and Functional Foods Institute, Université Laval, Québec, QC, Canada

Antonio Galvez Área de Microbiología, Departamento de Ciencias de la Salud, Facultad de Ciencias Experimentales, Edif. B3, Universidad de Jaén, Jaén, Spain

Sumathi Ganeshan Biology Programme, School of Distance Education, Universiti Sains Malaysia, Minden, Penang, Malaysia

Elisabeth Grohmann Division of Infectious Diseases, University Medical Center Freiburg, Freiburg, Germany

Fazil Hasan Department of Plant Protection, Faculty of Agricultural Sciences, Aligarh Muslim University, Aligarh, India

Mohammad Aamir Hussain Department of Biochemistry, Faculty of Life Sciences, Aligarh Muslim University, Aligarh, Uttar Pradesh, India

Hassan Mubarak Ishqi Department of Biochemistry, Faculty of Life Sciences, Aligarh Muslim University, Aligarh, Uttar Pradesh, India 
Surinder Kaur Department of Mycology \& Plant Pathology, Institute of Agricultural Sciences, Banaras Hindu University (BHU), Varanasi, Uttar Pradesh, India

INRS-ETE, Université du Québec, Québec, Canada

Saman Khan Molecular and Structural Biology Division, CSIR - Central Drug Research Institute, Lucknow, India

Alette A.M.Langenhoff Sub-department of Environmental Technology, Wageningen University, Wageningen, The Netherlands

Nelson Lima IBB - Biological Engineering Centre, Applied Mycology Group, University of Minho, Braga, Portugal

Showkat Ahmad Lone Biotechnology and Climate Change, National Research Centre on Plant Biotechnology, New Delhi, India

Department of Agricultural Microbiology, Faculty of Agricultural Sciences, Aligarh Muslim University, Aligarh, Uttar Pradesh, India

Abdul Malik Department of Agricultural Microbiology, Faculty of Agricultural Sciences, Aligarh Muslim University, Aligarh, Uttar Pradesh, India

Farhana Masood Department of Agricultural Microbiology, Faculty of Agricultural Sciences, Aligarh Muslim University, Aligarh, Uttar Pradesh, India

Farrakh Mehboob Laboratory of Microbiology, Wageningen University, Wageningen, The Netherlands

Ecotoxicology Research Programme, National Institute of Bio-remediation, National Agriculture Research Centre, Islamabad, Pakistan

Hideaki Nojiri Biotechnology Research Center, The University of Tokyo, Bunkyo-ku, Tokyo, Japan

Agricultural Bioinformatics Research Unit, The University of Tokyo, Bunkyo-ku, Tokyo, Japan

Nabil Ben Omar Área de Microbiología, Departamento de Ciencias de la Salud, Facultad de Ciencias Experimentales, Edif. B3, Universidad de Jaén, Jaén, Spain

Jasdeep Chatrath Padaria Biotechnology and Climate Change, National Research Centre on Plant Biotechnology, New Delhi, India

Leonel Pereira IBB - Biological Engineering Centre, Applied Mycology Group, University of Minho, Braga, Portugal

M.A. Pereira IBB - Institute for Biotechnology and Bioengineering, Centre of Biological Engineering, Universidade do Minho, Braga, Portugal

E.V. Pershina All-Russia Research Institute for Agricultural Microbiology, Saint-Petersburg, Russia

A.G. Pinaev All-Russia Research Institute for Agricultural Microbiology, Saint-Petersburg, Russia 
N.A. Provorov All-Russia Research Institute for Agricultural Microbiology, Saint-Petersburg, Russia

Sayeed Ur Rehman Department of Biochemistry, Faculty of Life Sciences, Aligarh Muslim University, Aligarh, Uttar Pradesh, India

Cledir Santos IBB - Biological Engineering Centre, Applied Mycology Group, University of Minho, Braga, Portugal

Tarique Sarwar Department of Biochemistry, Faculty of Life Sciences, Aligarh Muslim University, Aligarh, Uttar Pradesh, India

Gosse Schraa Laboratory of Microbiology, Wageningen University, Wageningen, The Netherlands

Masaki Shintani Department of Materials Science and Chemical Engineering, Faculty of Engineering, Shizuoka University, Shizuoka, Japan

Japan Collection of Microorganisms, RIKEN BioResource Centre, Tsukuba, Ibaraki 305-0074, Japan

Marta F. Simões IBB - Biological Engineering Centre, Applied Mycology Group, University of Minho, Braga, Portugal

D.P. Singh Department of Environmental Science, Babasaheb Bhimrao Ambedkar (Central) University, Lucknow, Uttar Pradesh, India

Jay Shankar Singh Department of Environmental Microbiology, Babasaheb Bhimrao Ambedkar (Central) University, Lucknow, Uttar Pradesh, India

Amit Kumar Sonkar Molecular and Structural Biology Division, CSIR - Central Drug Research Institute, Lucknow, India

Diana Sousa IBB - Institute for Biotechnology and Bioengineering, Centre of Biological Engineering, University of Minho, Braga, Portugal

Alfons J.M. Stams Laboratory of Microbiology, Wageningen University, Wageningen, The Netherlands

Centre of Biological Engineering, University of Minho, Braga, Portugal

Mohammad Tabish Department of Biochemistry, Faculty of Life Sciences, Aligarh Muslim University, Aligarh, Uttar Pradesh, India

Mausum Verma Institut de recherche et de développement en agroenvironnement inc. (IRDA), Québec, QC, Canada

Zuraini Zakaria Biology Programme, School of Distance Education, Universiti Sains Malaysia, Minden, Penang, Malaysia

Mohammad Zuhaib Department of Plant Protection, Faculty of Agriculture Sciences, Aligarh Muslim University, Aligarh, Uttar Pradesh, India 